\title{
FATORES DE RISCO PARA DOENÇA CARDIOVASCULAR E AVALIAÇÃO QUALITATIVA DA ALIMENTAÇÃO EM UNIVERSITÁRIOS
}

\author{
RISK FACTORS FOR CARDIOVASCULAR DISEASE AND QUALITATIVE EVALUATION \\ OF DIETARY INTAKE OF UNDERGRADUATE STUDENTS
}

\author{
Luana Romão Nogueira ${ }^{1}$ \\ Aline Veroneze de Mello ${ }^{1}$ \\ Rosana Farah Simony Lamigueiro Toimil ${ }^{2}$
}

Resumo: O consumo de alimentos ricos em carboidratos refinados, colesterol, pobres em fibras alimentares e com elevados teores de gorduras saturadas e trans aumentam o risco de desenvolvimento de DCVs. Por outro lado, a ingestão de carnes magras, frutas, verduras, legumes e alimentos integrais possui efeito protetor contra 0 processo aterosclerótico; por isso, é importante adequar a qualidade da alimentação para prevenir o risco cardiovascular. Portanto, os objetivos deste trabalho são: avaliar o consumo alimentar dos universitários e avaliar o acúmulo de gordura abdominal. Trata-se de estudo transversal, com universitários do município de São Paulo. A coleta de dados foi realizada por meio de um questionário que abordou dados pessoais, hábitos de vida, dados antropométricos referidos (altura e peso), circunferência de abdômen e Questionário de Frequência Alimentar. Foram avaliados 62 universitários, sendo a maioria do sexo feminino, com idade entre 19 e 41 anos, solteiros, sedentários, não-fumantes e não consumidores de bebidas alcoólicas regularmente. Não possuem doenças crônicas, porém apresentam histórico familiar de HAS e Diabetes Mellitus. A amostra apresentou média de IMC de $22,2 \mathrm{~kg} / \mathrm{m}^{2}$ e circunferência de cintura de 78,6 centímetros para as mulheres e 85,5 centímetros para os homens. Em relação ao consumo alimentar, os universitários apresentaram baixo consumo de leite desnatado, iogurte, peixes, frutos do mar, frutas, carne bovina frita, frango frito ou com pele, manteiga, margarina, refrigerantes, sucos artificiais, sanduíches, pizza e fast-food, bolos, bolachas, chocolates. Os universitários apresentaram poucos fatores de risco para DCVs: sedentarismo, baixo consumo de alimentos protetores e antecedentes familiares de doenças crônicas.

Palavras-chave: doenças cardiovasculares; fatores de risco; consumo de alimentos; estudantes.

\begin{abstract}
Consumption of foods high in refined carbohydrates, cholesterol, low in fiber, and high levels of saturated and trans fats increases the risk of developing CVD. On the other hand, intake of lean meats, fruits, vegetables, and wholefood have a protective effect against the atherosclerotic process. Thus, it is important to adjust the quality of food to prevent cardiovascular risk. The aims of this study were to evaluate dietary intake of undergraduate students and assess their accumulation of abdominal fat. This cross-sectional study involved students from São Paulo. Data collection was conducted through a questionnaire regarding personal details as well as habits, such as anthropometric data (height and weight), circumference of abdomen, and a Food Frequency Questionnaire. The study evaluated 62 students, mostly female, between 19 and 41 years old, single, sedentary, non-smoking, who do not regularly consume alcohol. They do not have chronic diseases, although they have both family history of hypertension and diabetes mellitus. The sample has an average Body Mass Index (BMI) of $22.2 \mathrm{Kg} / \mathrm{m}^{2}$ and a waist circumference of 78.6 centimeters for the women and 85.5 centimeters for the men. Concerning food consumption, the undergraduate students consume a low amount of skim milk, yogurt, fish, seafood, fruits, fried beef, and fried or skin-on chicken, butter, margarine, soft drinks, artificial juices, sandwiches,
\end{abstract}

\footnotetext{
1 Graduada em Nutrição - Universidade Presbiteriana Mackenzie - UPM, Brasil. E-mails: luanaromaon@hotmail.com; alinevm3@hotmail.com.

${ }^{2}$ Doutora em Ciências Médicas - Universidade Federal de São Paulo - Unifesp, Brasil. Docente da UPM, Brasil. E-mail: rosana.simony@mackenzie.br.
} 
pizza and fast food, cakes, cookies, chocolates. The undergraduate students presented a few risk factors for CVD: sedentary lifestyle, low consumption of protector foods, and family history of chronic diseases.

Keywords: cardiovascular diseases; risk factors; food consumption; students.

\section{INTRODUÇÃO}

O Brasil está entre os 10 países com maior número de mortes causadas por doenças cardiovasculares (DCV), sendo a principal razão de morte em mulheres e homens no país. São quase $30 \%$ de mortes todos os anos devido à doença (MANSUR; FAVARATO, 2012). Sua etiologia é multifatorial, contudo existem fatores que parecem influenciar mais fortemente a ocorrência da doença, sendo eles: tabagismo, hipertensão arterial, hipercolesterolemia, diabetes, obesidade e sedentarismo (GIROTTO et al.,1996).

Existem evidências de que o processo aterosclerótico, onde há deposição de lipídeos nas paredes dos vasos, inicia-se na infância e possui o tempo de latência muito longo (PETRIBÚ; CABRAL; ARRUDA, 2009). As mudanças de comportamento devido o ingresso no ensino superior podem agravar esse processo, uma vez que é comum nesta fase da vida a omissão de refeições, ingestão de alimentos pobres nutricionalmente, consumo de álcool, tabagismo e instabilidade psicossocial (VIEIRA et al., 2002).

O consumo de alimentos ricos em carboidratos refinados, colesterol, pobres em fibras alimentares e com elevados teores de gorduras saturadas e trans também eleva consideravelmente o risco de desenvolvimento de DCVs (SANTOS et al., 2006). Por outro lado a ingestão de carnes magras, frutas, verduras, legumes e alimentos integrais possui efeito protetor contra o processo aterosclerótico, por isso é de fundamental importância adequar a qualidade da alimentação para prevenir o risco cardiovascular (CHIARA; SICHIERI, 2001). A American Heart Association (AHA) elaborou um guia que enfatiza o consumo de vegetais, frutas e grãos integrais, confirmando a importância das fibras alimentares, antioxidantes e outras substâncias na prevenção e controle das DCVs. Recomenda ainda, a manutenção de peso saudável, auxiliado pela atividade física regular e consumo moderado de gorduras (ECKEL et al., 2013).

Considerando todos os aspectos aqui expostos, o presente estudo tem como finalidade avaliar a presença de fatores de risco cardiovascular em universitários adultos. Entre os objetivos específicos, pretende-se avaliar o consumo alimentar dos universitários e avaliar o acúmulo de gordura abdominal.

\section{METODOLOGIA}

\subsection{Tipo de estudo}

O presente estudo teve delineamento transversal. 


\subsection{Amostra}

A amostra foi composta por estudantes regularmente matriculados em uma universidade particular do município de São Paulo. Todos os participantes que aceitaram participar da pesquisa responderam ao questionário e assinaram o Termo de Consentimento Livre e Esclarecido (TCLE).

Por ser um estudo que envolve seres humanos, o projeto foi submetido e aprovado pelo Comitê de Ética em Pesquisa da Universidade Presbiteriana Mackenzie.

\subsection{Coleta de dados}

A coleta foi realizada por meio de um questionário que abordou dados sobre idade, sexo, estado civil, presença e histórico de doenças, atividade física, tabagismo, etilismo, dados antropométricos referidos (altura e peso), circunferência de abdômen (localizada na altura da cicatriz umbilical) aferida pelo pesquisador, utilizando-se uma fita métrica inelástica e flexível com precisão de $1 \mathrm{~mm}$ da marca Lemat® e o consumo alimentar foi avaliado por meio da aplicação de um Questionário de Frequência Alimentar adaptado do estudo "Desenvolvimento de um questionário de frequência alimentar para estudo de dieta e doença cardiovascular em estudantes universitários" do Congresso de Iniciação Científica da Universidade Federal de Pernambuco (LIMA, 2008).

\subsection{Análise de dados}

As variáveis coletadas foram tabuladas com o auxílio do software Microsoft Excel® 2010. Os resultados qualitativos foram analisados por meio da distribuição da frequência em número e porcentagem, e as variáveis quantitativas por medidas de posição e dispersão. Os resultados foram apresentados por meio de tabelas e gráficos.

A partir dos dados de peso e altura, foi calculado o Índice de Massa Corporal (IMC= peso/altura²), avaliado pela classificação proposta pela Organização Mundial da Saúde (WHO, 2004). A obesidade abdominal foi avaliada segundo as referências da IV Diretriz Brasileira Sobre Dislipidemias e Prevenção da Aterosclerose do Departamento de Aterosclerose da Sociedade Brasileira de Cardiologia (SBC, 2007).

\section{RESULTADOS E DISCUSSÃO}

O presente estudo avaliou 62 universitários adultos. A Tabela 1 mostra as principais características sócio-demográficas da amostra. Como pode ser observado, a maioria dos participantes do estudo eram do sexo feminino, com idades variando entre 19 a 41 anos e 93,6\% eram solteiros. Estudos demonstram que homens possuem maior risco de desenvolvimento de doenças cardiovasculares do que mulheres, uma vez que as 
manifestações desse tipo de doença progridem de forma acelerada após a quinta década nos homens, e após a sexta década em mulheres (VERAS et al., 2007). Em relação a doenças presentes, 90,3\% referiram não possuir nenhuma doença, entre aqueles que relataram, destacou-se a Diabetes Mellitus, apesar de apresentar valor percentual de apenas $4,8 \%$.

Tabela 1 - Principais características sócio-demográficas e clínicas de universitários. São Paulo, 2015

\begin{tabular}{|c|c|}
\hline Variáveis & Resultados \\
\hline Sexo (M/F) & $6 / 56$ \\
\hline Idade (anos) & $23,1 \pm 4,3$ \\
\hline Estado Civil [n(\%)] & $4(6,4)$ \\
Casado & $58(93,6)$ \\
Solteiro & $56(90,3)$ \\
Doenças referidas [n(\%)] & $3(4,8)$ \\
Sem doença referida & $1(1,6)$ \\
Diabetes Mellitus & $1(1,6)$ \\
Asma & $1(1,6)$ \\
Intolerância à lactose & \\
Síndrome do intestino irritável &
\end{tabular}

Ao analisar o histórico familiar, $66 \%$ relataram possuir familiares próximos com algum tipo de doença crônica, sendo que a mais citada foi a Hipertensão Arterial Sistêmica $(38,7 \%)$, seguida da Diabetes Mellitus $(20,9 \%)$, além de câncer $(3,2 \%)$ e asma $(3,2 \%)$. Em estudo realizado por Correia, Cavalcante e Santos (2010), que também analisaram o histórico familiar de universitários, a HAS também foi a doença mais prevalente $(63,6 \%)$, seguida de Diabetes Mellitus (54,5\%), o que demonstra um resultado semelhante ao do presente estudo.

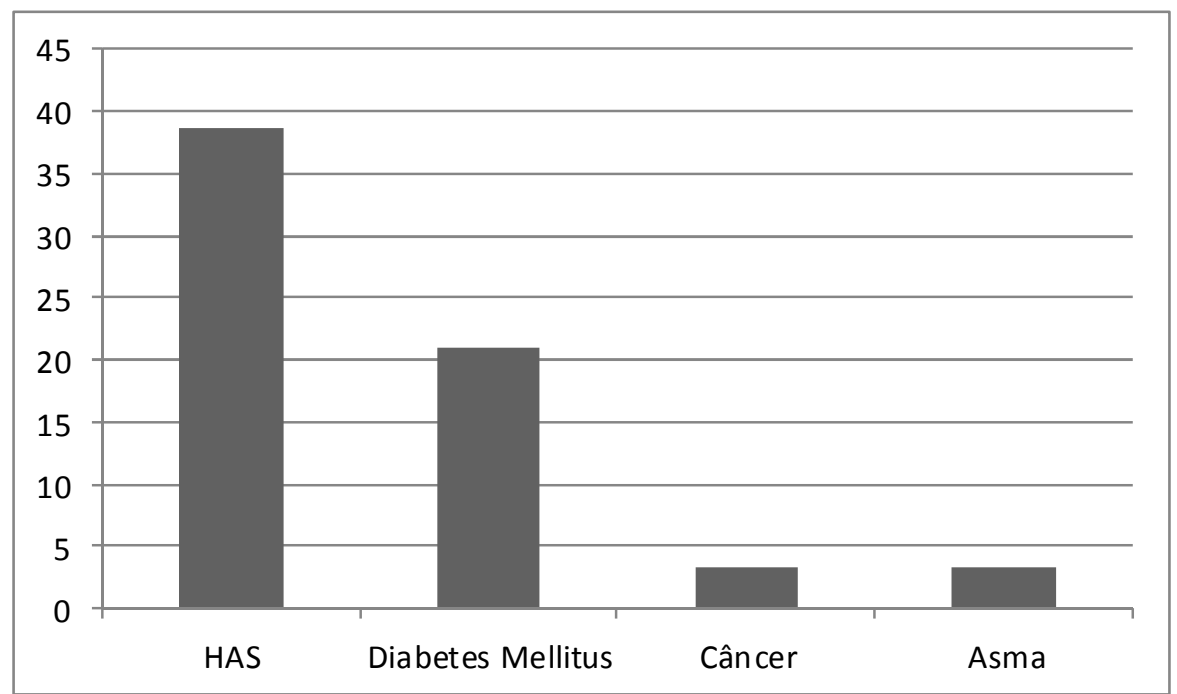

Figura 1 - Distribuição em percentual do histórico familiar de doenças dos universitários. São Paulo, 2015. 
Com relação à atividade física, 51,6\% dos universitários eram sedentários. A Figura 2 aponta que a amostra referiu como principais atividades físicas diárias a caminhada, a musculação, a natação, a dança e o futebol. Resultado semelhante foi encontrado por Marcondelli, Costa e Schmitz (2008), em que 65,5\% da amostra composta por universitários da área da saúde eram sedentários. A atividade física regular é uma medida auxiliar para o controle das dislipidemias. O exercício físico aeróbio reduz níveis plasmáticos de triglicérides, evitando sua deposição arterial, e aumenta os níveis de HDL, responsável por realizar o transporte inverso do colesterol, ou seja, dos vasos sanguíneos para o fígado (SBC, 2007).

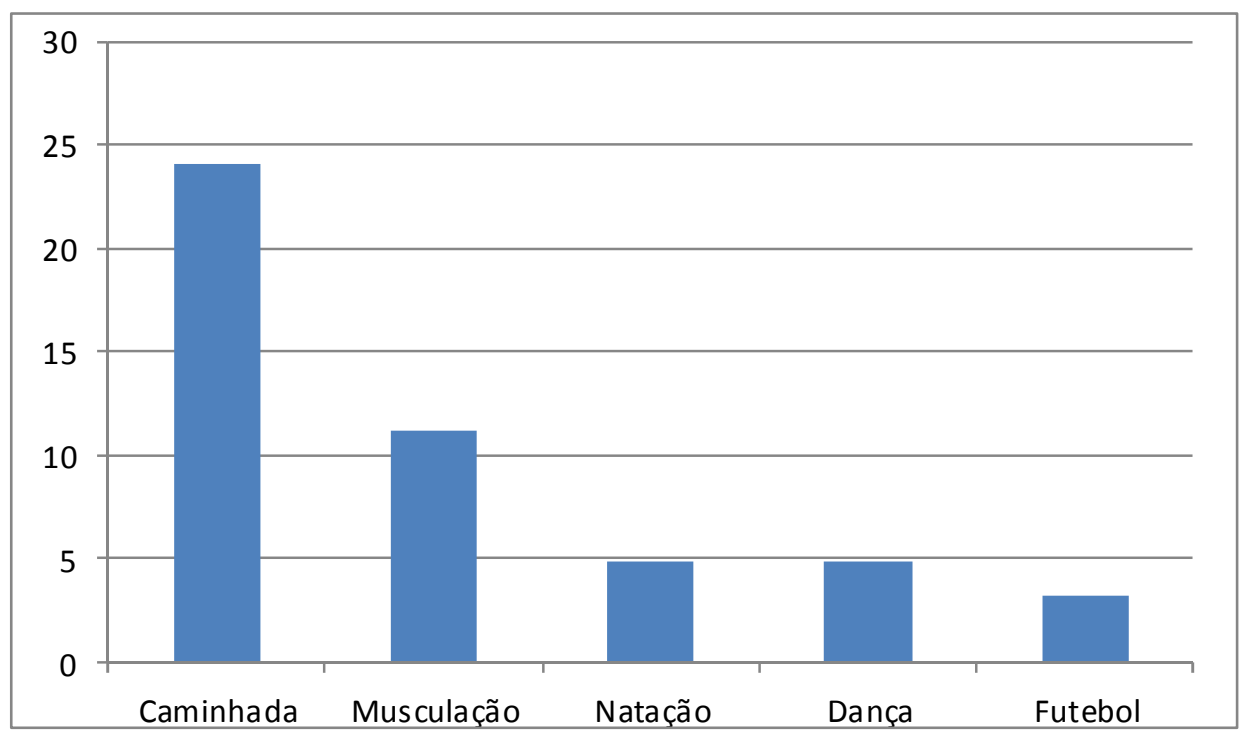

\section{Figura 2 - Distribuição em percentual dos tipos de atividade física realizada por universitários. São Paulo, 2015.}

Quando observadas as características relacionadas ao estilo de vida, 6,4\% referiram o hábito de fumar, e 24,2\% relataram que consomem bebidas alcoólicas regularmente.

Em relação ao estado nutricional, segundo IMC da amostra (Tabela 2), a média foi de $22,2 \mathrm{~kg} / \mathrm{m}^{2}$ classificados como eutróficos, ou seja, peso adequado para altura. Contudo, ao analisar separadamente, $6,4 \%$ encontram-se abaixo do peso e $11,3 \%$ com sobrepeso. Em estudo realizado em 2007 para o levantamento de fatores de risco para doenças crônicas em universitários, nenhum dos participantes estava abaixo do peso, 25\% estavam com sobrepeso e 5,9\% encontravam-se em obesidade grau 1 (VERAS et al., 2007). As diferenças encontradas podem ser justificadas, uma vez que o estudo comparativo foi realizado em Fortaleza e predominavam participantes do sexo masculino. Em outro estudo de 2012, realizado com estudantes da área de saúde de uma universidade particular, em São José dos Campos, 6,5\% da amostra encontravam-se em baixo peso e $21,3 \%$ em sobrepeso, resultados semelhantes aos encontrados neste trabalho (SOAR; SILVA; LIRA, 2012). 


\section{Tabela 2 - Distribuição percentual do índice de massa corpórea (IMC) em universitários de ambos os gêneros. São Paulo, 2015}

\begin{tabular}{lc}
\hline \multicolumn{1}{c}{$\begin{array}{c}\text { Classificação do estado } \\
\text { nutricional }\end{array}$} & N (\%) \\
\hline Baixo peso & $4(6,4)$ \\
Eutrófico & $51(82,3)$ \\
Sobrepeso & $7(11,3)$ \\
Obesidade & - \\
\hline
\end{tabular}

Para a análise da gordura abdominal, foi realizada a circunferência da cintura e a amostra composta por mulheres apresentou média de 78,6 centímetros e os homens 85,5 centímetros. A Diretriz Brasileira Sobre Dislipidemias e Prevenção da Aterosclerose do Departamento de Aterosclerose da Sociedade Brasileira de Cardiologia considera como risco para complicações metabólicas, circunferência de cintura maior ou igual a 80 centímetros para mulheres e, maior ou igual a 94 centímetros para homens. Entretanto, a amostra estudada não apresentou risco metabólico. A obesidade abdominal ou central está associada a alterações no perfil lipídico, bem como aumento da pressão arterial, hiperinsulinemia, e esses são fatores de risco para diabetes mellitus tipo 2, doenças cardiovasculares e resistência à insulina (SANTOS et al., 2013).

Com relação ao consumo alimentar dos universitários (Tabela 3), alimentos protetores para DCVs como leite desnatado, iogurte, peixes, frutos do mar e frutas apresentaram consumo baixo, uma vez que, para a maioria desses alimentos, os participantes referiram consumir menos de 4 vezes na semana. As frutas são alimentos fonte de fibras solúveis, que reduzem o tempo de trânsito intestinal e absorção do colesterol (SBC, 2007). O azeite que também é um alimento protetor apresentou maior consumo, cerca de 40,3\% utilizamno todos os dias nas refeições. Resultado semelhante ao encontrado por Neumann, Shirassu e Fisberg (2006), em que $36,5 \%$ da amostra composta por funcionários públicos referiram consumo diário de azeite. 
Tabela 3 - Consumo alimentar de universitários. São Paulo, 2015

\begin{tabular}{|c|c|c|c|c|}
\hline & $\begin{array}{c}\text { Nunca/ } \\
\text { raramente }\end{array}$ & $<4 x /$ sem & $\geq 4 x /$ sem & 1 ou mais/dia \\
\hline Leite integral & 42 & 9 & 2 & 9 \\
\hline Leite desnatado & 32 & 15 & 8 & 7 \\
\hline logurte & 19 & 20 & 12 & 11 \\
\hline Queijos amarelos & 9 & 22 & 23 & 8 \\
\hline Carne bovina cozida & 7 & 30 & 16 & 9 \\
\hline Carne bovina frita & 37 & 19 & 6 & - \\
\hline Frango cozido sem pele & 11 & 36 & 13 & 2 \\
\hline Frango com pele ou frito & 39 & 13 & 9 & 1 \\
\hline Peixes/frutos do mar & 24 & 26 & 9 & 3 \\
\hline Alimentos embutidos & 18 & 27 & 12 & 5 \\
\hline Feijão & 8 & 14 & 28 & 12 \\
\hline Salada crua & 3 & 12 & 28 & 19 \\
\hline Banana & 6 & 27 & 18 & 11 \\
\hline Acerola/laranja & 13 & 27 & 19 & 3 \\
\hline Maracujá & 28 & 25 & 7 & 2 \\
\hline Maçã & 14 & 34 & 10 & 4 \\
\hline Mamão & 27 & 21 & 11 & 3 \\
\hline Goiaba & 33 & 20 & 8 & 1 \\
\hline Melancia & 20 & 33 & 6 & 3 \\
\hline Uva & 18 & 35 & 5 & 4 \\
\hline Abacaxi & 23 & 25 & 12 & 2 \\
\hline Sucos naturais de frutas & 9 & 21 & 21 & 11 \\
\hline Arroz/macarrão & 6 & 12 & 10 & 34 \\
\hline Arroz/bolacha integral & 20 & 14 & 12 & 16 \\
\hline Bolacha/biscoito/bolo & 18 & 27 & 10 & 7 \\
\hline Batata & 8 & 37 & 14 & 3 \\
\hline Óleos & 16 & 14 & 10 & 22 \\
\hline Margarina & 26 & 17 & 6 & 13 \\
\hline Manteiga & 40 & 17 & 3 & 2 \\
\hline Maionese & 38 & 16 & 3 & 5 \\
\hline Azeite & 7 & 15 & 15 & 25 \\
\hline Açúcar & 20 & 23 & 10 & 9 \\
\hline Balas/doces & 14 & 26 & 16 & 6 \\
\hline Achocolatado/chocolate & 18 & 15 & 17 & 12 \\
\hline Refrigerantes & 22 & 28 & 10 & 2 \\
\hline Café & 34 & 19 & 6 & 3 \\
\hline Suco artificial & 31 & 15 & 10 & 6 \\
\hline Sanduíche/pizza/fast-food & 15 & 35 & 9 & 3 \\
\hline Ketchup/mostarda & 43 & 15 & 2 & 2 \\
\hline
\end{tabular}


Sobre os alimentos de risco, como carne bovina frita, frango frito ou com pele, manteiga, refrigerantes, sucos artificiais, sanduíches, pizza e fast-food, também apresentaram consumo considerado como baixo. Nota-se que os participantes do estudo preferem carne bovina cozida em relação à frita, contudo, mesmo a carne bovina cozida não apresentou consumo alto, pois apenas $14,5 \%$ consomem o alimento todos os dias. No presente estudo, consideram-se esses resultados como positivos, dado que a Sociedade Brasileira de Cardiologia (2007) recomenda a redução da ingestão de gordura animal, pois influenciam, diretamente, os níveis lipídicos plasmáticos, em especial à colesterolemia.

A maioria dos pesquisados $(35,4 \%)$ utiliza óleos vegetais todos os dias nas preparações dos alimentos. A substituição de ácidos graxos saturados por ácidos graxos insaturados, presentes nos peixes, óleos vegetais, oleaginosas reduz o colesterol total e o LDL (SBC, 2007).

Alimentos como bolos, bolachas, chocolates, margarina, que são fonte de ácidos graxos trans também apresentaram consumo considerado baixo. Esse tipo de ácido graxo aumenta o LDL e reduz o HDL; por isso, esses alimentos são considerados de risco para DCVs (SBC, 2007).

O presente estudo contribuiu para maior conhecimento dos hábitos alimentares, hábitos de vida e alguns fatores de risco para DCV, em universitários, contudo, apresenta fatores limitantes, como a utilização de apenas dois instrumentos para avaliação da composição corporal (IMC e circunferência da cintura). Além disso, o Questionário de frequência alimentar é um método que depende da memória dos indivíduos e alguns desses tendem a omitir o consumo de alguns tipos de alimentos e reduzir quantidades.

\section{CONCLUSÃO}

A maioria dos universitários estudados é do sexo feminino, com idades variando entre 19 e 41 anos, solteiros, sedentários, não-fumantes e não consumidores de bebidas alcoólicas regularmente. Não possuem doenças crônicas, contudo a maioria apresenta histórico familiar de HAS, seguida de Diabetes Mellitus.

Em relação ao estado nutricional, a amostra apresenta-se eutrófica e não apresenta gordura abdominal aumentada de acordo com a circunferência da cintura.

Sobre o consumo alimentar, os universitários apresentaram baixo consumo de leite desnatado, iogurte, peixes, frutos do mar, frutas, carne bovina frita, frango frito ou com pele, manteiga, margarina, refrigerantes, sucos artificiais, sanduíches, pizza e fast-food, bolos, bolachas, chocolates.

De modo geral, os universitários apresentaram poucos fatores de risco para DCV, como o sedentarismo, baixo consumo de alimentos protetores e antecedentes familiares de doenças crônicas. 


\section{REFERÊNCIAS BIBLIOGRÁFICAS}

CHIARA, V. L.; SICHIERI, R. Consumo alimentar em adolescentes. Questionário simplificado para avaliação de risco cardiovascular. Arq. Bras. Cardiol. São Paulo, v. 77, n. 4, p. 332-336, 2001.

CORREIA, B. R.; CAVALCANTE, E.; SANTOS, E. A prevalência de fatores de risco para doenças cardiovasculares em estudantes universitários. Rev. Bras. Clin. Med., Santos, n. 8, p. 25-29, 2010.

ECKEL, R. H. et al. AHA/ACC Guideline on Lifestyle Management to Reduce Cardiovascular Risk. Journal of the American Heart Association. p. 1-45, 2013.

GIROTTO, C. A. et al. Prevalencia de factores de riesgo cardiovascular en ingresantes universitários. Rev. Saúde Pública, São Paulo, v. 30, n. 6, p. 576-586, 1996.

LIMA, R. F.; CABRAL, P. C. Questionário Desenvolvimento de um questionário de frequência alimentar para estudo de dieta e doença cardiovascular em estudantes universitários. In.: CONGRESSO DE INICIAÇÃO CIENTÍFICA DA UFPE, 15., 2008, Recife. Anais ... Recife: UFPE, $2008 . \quad$ Disponível em: <https://www.ufpe.br/conic/images/stories/anais/2008/conic/pibic/40/074051105SCPO.pdf>. Acesso em: 23 abr. 2015.

MANSUR, A. P.; FAVARATO, D. Mortalidade por doenças cardiovasculares no Brasil e na região metropolitana de São Paulo: atualização 2011. Arq. Bras. Cardiol., São Paulo, v. 99, n. 2, p. 755-761, 2012.

MARCONDELLI, P.; COSTA, T. H. M.; SCHMITZ, A. S. Nível de atividade física e hábitos alimentares de universitários do $3^{\circ}$ ao $5^{\circ}$ semestres da área da saúde. Rev. Nutr., Campinas, v. 21, n. 1, p. 39-47, 2008.

NEUMANN, A. I. C. P.; SHIRASSU, M. M.; FISBERG, R. G. Consumo de alimentos de risco e proteção para doenças cardiovasculares entre funcionários públicos. Rev. Nutr. Campinas, v. 19, n. 1, p. 19-28, 2006.

PETRIBÚ, M. M. V.; CABRAL, P. C.; ARRUDA, I. K. G. Estado nutricional, consumo alimentar e risco cardiovascular: um estudo em universitários. Rev. Nutr., Campinas, v. 22, n. 6, p. 837-846, 2009.

SANTOS, C. R. B. et al. Fatores dietéticos na prevenção e tratamento de comorbidades associadas à síndrome metabólica. Rev. Nutr., Campinas, v. 19, n. 3, p. 389-401, 2006.

SANTOS, H. C. M. et al. Síndrome metabólica e outros fatores de risco para doenças cardiovascular em população de obesos. Rev. Bras. Cardiol. Rio de Janeiro, v. 26, n. 3, p. 442-449, 2013. 
SOAR, C.; SILVA, P. S.; LIRA, J. G. Consumo alimentar e atividade física de estudantes universitários da área da saúde. Rev. Univap, São José dos Campos, v. 18, n. 31, p. 41-47, 2012.

SOCIEDADE BRASILEIRA DE CARDIOLOGIA (SBC). IV Diretriz Brasileira sobre Dislipidemias e Prevenção da Aterosclerose: Departamento de Aterosclerose da Sociedade Brasileira de Cardiologia. 2007. Disponível em: <http://publicacoes.cardiol.br/consenso/2007/IV diretriz DA.asp>. Acesso em: 22 abr. 2013.

VERAS, V. S. et al. Levantamento dos fatores de risco para doenças crônicas em universitários. RBPS, Fortaleza, v. 20, n. 3, p. 168-172, 2007.

VIEIRA, V. C. R. et al. Perfil socioeconômico, nutricional e de saúde de adolescentes recémingressos em uma universidade pública brasileira. Rev. Nutr. Campinas, v. 15, n. 3, p. 273282, 2002.

WORLD HEALTH ORGANIZATION (WHO). Body Mass Index classification. 2004. Disponível em: <http://apps.who.int/bmi/index.jsp?introPage=intro 3.html>. Acesso em: 17 abr. 2013. 